\title{
Retraction Note to: Randomized clinical trial of arginine-supplemented enteral nutrition versus standard enteral nutrition in patients undergoing gastric cancer surgery
}

\author{
Hongyan Zhao $\cdot$ Hongying Zhao $\cdot$ Yu Wang $\cdot$ \\ Huang Jing $\cdot$ Qian Ding $\cdot$ Jun Xue
}

Published online: 20 January 2015

(C) Springer-Verlag Berlin Heidelberg 2015

\section{Retraction Note to: J Cancer Res Clin Oncol (2013) 139:1465-1470 \\ DOI 10.1007/s00432-013-1466-5}

Our paper entitled "Randomized clinical trial of argininesupplemented enteral nutrition versus standard enteral nutrition in patients undergoing gastric cancer surgery" reported that arginine-supplemented enteral nutrition significantly improved long-term survival and restored immunity in malnourished gastric cancer. However, recently we checked our data again and found there was an obvious difference between experimental and control groups in the tumor status at the beginning of the trial. In addition, the sample size was not calculated, which might be not enough to reach a significant power. Thus, the trial might be biased and we, the authors, believe that the most responsible course of action is to retract our paper. We deeply regret this circumstance and apologize to the community.

The online version of the original article can be found under doi:10.1007/s00432-013-1466-5.

Hongyan Zhao

Department of Intensive Care Unit, Daqing Oilfield General

Hospital, Daqing 163001, China

Hongying Zhao

Department of Oncology, Daqing Longnan Hospital,

Daqing 163453, China

\section{Y. Wang}

Department of General Surgery, Daqing Longnan Hospital,

Daqing 163453, China

H. Jing $\cdot$ Q. Ding $\cdot$ J. Xue $(\varangle)$

Cancer Center, Union Hospital, Tongji Medical College,

Huazhong University of Science and Technology, Wuhan 430022,

China

e-mail: xuejuntju@163.com 